\title{
LA TIPOLOGÍA EN LA VIVIENDA COMO PRECEDENTE SUSTENTABLE
}

\section{Selenne Galeana Cruz ${ }^{1}$}

Maestría en Procesos y Expresión Gráfica en la Proyectación Arquitectónica Urbana. Universidad de Guadalajara.

Palabras Clave: Vivienda adecuada, Transformación espacial, Tipología, función, forma.

Resumen: El presente artículo intenta realizar una aportación para el análisis tipológico de la vivienda, en particular a la vivienda social. Esta intención se enmarca en el análisis de las características de la vivienda adecuada, que se inscribe como uno de los Objetivos del Milenio propuesto por la ONU, cuya evaluación y monitoreo ha sido responsabilidad del Programa UNHABITAT.

Para su tratamiento se ha elegido un enfoque Pospositivista (los principales exponentes son Wilhelm Dilthey y Willian James), es decir experimental, cuantitativo, causal y comparativo de una realidad conocida en términos de probabilidad.

Para abordarlo se propone un Instrumento de Análisis Gráfico Espacial-IAGE, partiendo para su construcción de la transformación espacial como categoría de análisis estrechamente vinculada con el referente sustentable físico-espacial a través del concepto de tipología. Por lo tanto este instrumento se estructura en tres dimensiones: formal, funcional y sociopolítica, las cuales estarán vinculadas a variables e indicadores desarrollados a partir de la composición de preceptos conceptuales y herramientas gráficas. Se deduce que dicha herramienta de limita a aspectos cuantitativos, sin embargo se considera pertinente la incorporación del enfoque cualitativo en estudios posteriores, de tal forma que sea posible realizar un maclaje interpretativo objetivo y subjetivo.

\section{Contexto general}

El tema de la vivienda como objeto de análisis ha sido recurrente, sobre todo a partir la Revolución Industrial, periodo en el que se desarrollaron y difundieron una importante cantidad de estudios y manifiestos que establecían el acceso a la vivienda como un derecho social. Desde entonces y hasta la actualidad, el acceso a una vivienda adecuada se ha convertido en tema abordado tanto por organismos internacionales, especialistas y técnicos, como por las estructuras de gobierno. Según Suárez el derecho a una vivienda adecuada es la piedra angular de la Estrategia Mundial de la Vivienda:

"El derecho a una vivienda adecuada está reconocido universalmente por la comunidad de países... Todos los países, sin excepción, tienen algún tipo de obligación con respecto al sector de la vivienda, como lo

\footnotetext{
${ }^{1}$ Contacto: arquisel@hotmail.com
} 
demuestra la creación de ministerios u organismos de la vivienda, la asignación de fondos al sector de la vivienda y las correspondientes políticas, programas y proyectos... todos los ciudadanos de todos los Estados, por pobres que puedan ser, tienen derecho a esperar que sus gobiernos se preocupen de sus necesidades en materia de vivienda y que acepten una obligación fundamental de proteger y mejorar las casas y los barrios en lugar de perjudicarlos o destruirlos." (SUÁREZ, FRANCISCO, 2006; INTERNET)

Lo que es cierto es que el acceso a la vivienda adecuada es un tema fundamental para lograr lo que Gaja ha denominado sustentabilidad integral (Gaja, 2005) ${ }^{2}$, entendida como una categoría que implica tres dimensiones necesarias para lograr el desarrollo equitativo de los territorios: ambiental, económica y social. En el ámbito internacional el acceso a la vivienda adecuada forma parte de la agenda de la ONU y ha sido considerado entre los Objetivos de Desarrollo del Milenio (2000) y en el Programa UN-HÁBITAT (2002).

Para satisfacer el derecho a la vivienda, se especifica que los países miembros deben atender sus obligaciones en la materia a partir de la creación de programas o estrategias y proporcionando de manera equitativa, con relación a sus necesidades, recursos financieros a través de instancias u organismos establecidos para este propósito. La Comisión de Asentamientos Humanos y la Estrategia Mundial de la Vivienda hasta el Año 2000, establece en su párrafo 5 el concepto de vivienda adecuada:

“... significa disponer de un lugar donde poderse aislar si se desea, espacio adecuado, seguridad adecuada, iluminación y ventilación adecuadas, una infraestructura básica adecuada y una situación adecuada en relación con el trabajo y los servicios básicos, todo ello a un costo razonable". (WWW.ONU.ORG.MX; Abril 2006).

En este sentido, la Agenda Hábitat en su capítulo referido al Derecho de vivienda especifica dos indicadores clave para evaluar si una vivienda es adecuada: Estructuras durables (Clave1) y Área suficiente para vivir (Clave 2). Estos indicadores, el primero de orden cualitativo y el segundo cuantitativo, están relacionados con la conceptualización espacial de las necesidades del usuario de la vivienda, sin embargo son, desde nuestro punto de vista, insuficientes para evaluar si la vivienda es o no adecuada, considerando otros aspectos tipológicos y de índole cultural, geográfico-físico, etc.

Para aplicar los indicadores mencionados anteriormente, el programa UN-HÁBITAT ${ }^{3}$ ha establecido una estrategia de monitoreo, que consiste en la instalación de una red de Observatorios Urbanos en diferentes escalas (internacional, nacionales, locales), cuyo objetivo es evaluar indicadores propuestos por diversos especialistas, para posteriormente lograr interpretarlas en las políticas urbanas y estrategias de intervención sustentables. Así también Edwards Brian agrega: “...diseñar de forma sostenible también significa crear espacios que

\footnotetext{
${ }^{2}$ Gaja Díaz, Fernando. Revolución Informacional, crisis ecológica y urbanismo. Guadalajara, Jalisco, México: Editorial Universidad de Guadalajara, 2005, 2da. Edición, p. 205.

${ }^{3}$ Programa de Asentamientos Humanos de las Naciones Unidas (UN-HABITAT), 2003. (en línea).Mayo 2003. (citado 8 febrero 2008). P.24. Disponible en

Internet:http://www.unhabitatrolac.org/observatorios_urbanos.asp?id_secao=14\&id_idioma=2\#294.
} 
sean saludables, viables económicamente y sensibles a las necesidades sociales. Por si solo, un diseño responsable desde el punto de vista energético es de escaso valor..." (EDWARDS BRIAN, 2004:02)

Si bien los organismos internacionales han generado estrategias importantes respecto de la dotación de la vivienda adecuada, en México los programas se limitan a desarrollar programas para la producción masiva de viviendas que no cumplen con los requerimientos mínimos de adecuación para ser habitadas: sus espacios son excesivamente reducidos y/o no atienden las necesidades de los núcleos familiares predominantes, no responden a las condiciones geográfico-físicas y culturales de cada región, entre otros aspectos.

Por otro lado, se prevé que en el siglo XXI la sociedad será predominantemente urbana lo que significa que la demanda de vivienda en las ciudades, sobre todo en las del mundo menos desarrollado, seguirá incrementándose:

"...en la actualidad, la mitad de todos los seres humanos reside en zonas urbanas. De ellos la cuarta parte, aproximadamente vive en ciudades de más de un millón de habitantes, la mitad en megaciudades de más de ocho millones de habitantes. La presión mundial sobre el medio ambiente se dejará sentir primero en esas megaciudades, como Tokio, Ciudad de México y Sao Paulo." (EDWARDS, BRIAN, 2004:02)

Según datos emitidos por la ONU en América Latina y el Caribe (LAC) la mayor parte de la población reside en ciudades o asentamientos urbanos, a continuación en el cuadro 1 se proporciona la base de datos correspondiente al crecimiento de la población mundial en diversos periodos:

\begin{tabular}{|l|c|c|c|c|c|}
\hline \multicolumn{6}{|c|}{ Cuadro 1. Base de datos sobre el crecimiento de población mundial. } \\
\hline & \multicolumn{7}{|l|}{ Población Urbana } & $\begin{array}{c}\text { Tasa de crecimiento anual de la } \\
\text { población urbana (\%) }\end{array}$ \\
\hline & $\mathbf{2 0 0 0}$ & $\mathbf{2 0 1 5}$ & $\mathbf{2 0 3 0}$ & $\mathbf{2 0 0 0 - 2 0 1 5}$ & $\mathbf{2 0 1 5 - 2 0 3 0}$ \\
\hline Argentina & 89.9 & 92.6 & 93.9 & 1.3 & 0.9 \\
\hline Brasil & 81.3 & 86.5 & 88.9 & 1.5 & 1.0 \\
\hline Chile & 85.7 & 88.7 & 90.7 & 1.3 & 1.0 \\
\hline Colombia & 73.9 & 79.1 & 83.0 & 2.0 & 1.4 \\
\hline Cuba & 75.3 & 78.5 & 82.3 & 0.5 & 0.4 \\
\hline México & 74.4 & 77.9 & 81.9 & 1.6 & 1.2 \\
\hline $\begin{array}{l}\text { Fuente: PNUMA, Perspectivas del Medio Ambiente Urbano en América Latina y el Caribe, p.24 (2004). } \\
\text { (www.onu.org.mx) }\end{array}$
\end{tabular}

En América Latina y en particular en México, el acceso a la vivienda adecuada está vinculado a la formación de grandes asentamientos urbanos y al problema de la pobreza asentada principalmente en ciudades. En México, actualmente, más del $75 \%$ de la población habita en ciudades y se prevé que para el año 2030 este porcentaje rebase el $80 \%$. Si a este dato sumamos la población que vive en condiciones de pobreza, que en México en el año 2007 alcanzó la cifra de 44.7 millones de mexicanos en situación de pobreza patrimonial, es decir, que no cuentan con un ingreso suficiente para satisfacer sus necesidades esenciales y 14.4 
millones en pobreza alimentaria ${ }^{4}$, el escenario futuro no se vislumbra alentador, ya que en las ciudades se sumarán grandes sectores de pobreza, hábitats marginales, legales e ilegales, que ponen en un frágil equilibrio el desarrollo futuro de nuestra sociedad en un marco de equidad democrática.

Asimismo influye la transición democrática en la que están inmersos un importante número de países de nuestro continente y la corrupción como un problema íntimamente vinculado a sus estructuras de gobierno en todos sus niveles.

La ONU propone aplicar políticas de vivienda a partir de estrategias locales o acción municipal, adoptando ordenanzas y marcos regulatorios en asuntos sociales y ambientales que se ajusten a cada realidad específica, de esta forma ha otorgado la responsabilidad al gobierno de cada país miembro de gestionar su hábitat.

Si bien el problema del acceso a la vivienda hace referencia a una multitud de actores y factores de todo tipo, económicos, políticos, etc., la situación marginal en la que habita un alto porcentaje de la población hace de este un tema fundamental en la acción de los gobiernos nacionales y locales para promover programas oficiales de dotación de vivienda a los sectores sociales vulnerables. En México, estos programas tienen su origen en la Ley sobre Planeación General de la República (1930), teniendo como objetivo primordial coordinar y dirigir las actividades de las dependencias gubernamentales para obtener progresos en el desarrollo urbano y constructivo del país de acuerdo a las características de cada contexto como topografía, clima, población, historia, economía y tradiciones. A partir de entonces se crearon organismos e instancias con el fin de delimitar las directrices de las Secretarías de Estado y apoyar el desarrollo de los diferentes sectores. ${ }^{5}$

Consecuentemente surgieron diversos organismos como el Instituto Mexicano del Seguro Social (IMSS-1942), el Banco Nacional Hipotecario Urbano y de Obras Públicas (BANOBRAS1947) y el Instituto Nacional de Vivienda (INV-1954). Sin embargo fue hasta 1950 cuando el Estado empezó a asumir con mayor responsabilidad a la demanda de viviendas a través de organismos públicos y entregados en renta a los usuarios para el sector de escasos recursos. La intervención del Estado pretendió crecer de manera ilimitada reflejándose en la creación del Fondo Nacional de Vivienda (1972). Así surgieron 3 fondos representativos: INFONAVIT (Instituto del Fondo Nacional de la Vivienda para los Trabajadores), destinada a proporcionar vivienda a los trabajadores de empresas privadas, FOVISSSTE (Fondo de Vivienda del ISSSTE) para los trabajadores del estado, FOVIMI -ISSFAM (Fomento de Vivienda Militar) para las Fuerzas Armadas.

\footnotetext{
${ }^{4}$ CONEVAL, 2007. Comunicado Núm. 002/007, (citado el 09 febrero del 2008). (en línea). Disponible en internet: http://www.sedesol.gob.mx/archivos/0/File/Prog_Sectorial_WEB.pdf.1) Pobreza alimentaria: se refiere a las personas cuyo ingreso es menor al necesario para cubrir las necesidades de alimentación correspondientes a los requerimientos establecidos en la canasta alimentaria INEGI-CEPAL. 2) Pobreza de capacidades: se refiere a las personas cuyo ingreso es menor al necesario para cubrir el patrón de consumo básico de alimentación, salud y educación. Incluye a las personas en condiciones de pobreza alimentaria. 3) Pobreza patrimonial: se refiere a las personas cuyo ingreso es menor al necesario para cubrir el patrón de consumo básico de alimentación, vestido y calzado, vivienda, salud, transporte público y educación. Incluye a las personas en condiciones de pobreza alimentaria y de capacidades. ${ }^{5}$ Red latinoamericana de información en ordenamiento territorial. La ordenación del territorio (en línea). Septiembre 2004, (citado el 05 de febrero 2008). Disponible en internet: http://reliot.ine.gob.mx/otsedesol.html.
} 
Fue hasta los años 70 cuando se institucionalizó la planeación urbana con la publicación de la Ley General de Asentamientos Humanos en 1976 y con el primer Plan Nacional de Desarrollo Urbano en 1978. Este último con el objetivo principal de alcanzar un desarrollo urbano más equilibrado en el territorio y ordenado al interior de los centros de población, lo cual se vio reflejado en el Programa Nacional de Desarrollo Urbano y Vivienda 1984-1988, cuyos objetivos estuvieron relacionados con las políticas de adquisición y fomento de la vivienda para propiciar una mejor calidad de vida. Desde entonces los Planes de Desarrollo con sus respectivos Programas de Vivienda se han desarrollado en cada sexenio presidencial, desde entonces. Uno de los cambios más significativos sobre el tema, se produjo en 1992, a partir de la modificación de la Ley Orgánica de la Administración Pública Federal, con el propósito de conformar la Secretaría de Desarrollo Social-SEDESOL con atribuciones en materia de desarrollo urbano y ordenación de territorio. Consecuentemente se creo el Programa Nacional de Solidaridad-PRONASOL, el cual tenía como objetivo atender tanto a la población rural como a la urbana, en condiciones de pobreza extrema. En materia de vivienda, se pretendió atender principalmente lo referente a la dotación de servicios como: drenaje, agua, electricidad y pavimentación.

De esta forma la atención al problema de la vivienda en nuestro país ha contribuido a conformar extensos territorios de marginalidad, ya que la construcción de los espacios sostenibles parece ser un atributo vinculado al hábitat de un reducido sector privilegiado, que se apropia del patrimonio natural y paisajístico de los territorios urbanos del siglo XXI.

La política actual en materia de vivienda social ha cedido la producción de este bien a promotores inmobiliarios que especulan con esta problemática, generando problemas de salud y riesgo al producir viviendas en condiciones infrahumanas conformando paisajes deprimidos que arrastran consigo profundos problemas sociales y de salud: su ubicación es inadecuada ya que se establecen en los sectores de menos plusvalía en el territorio urbano, vinculados generalmente a zonas en condiciones de riesgo; la dimensión y confort (iluminación, ventilación, clima, etc.) de los espacios interiores no resuelven el problema de habitar ni en su concepción básica; la calidad de los materiales y sistemas constructivos están en función del costo mínimo y por lo tanto carecen de los estándares mínimos; Los equipamientos recreativos, escolares y de salud y son inexistentes, insuficientes o inadecuados, etc.

...el espacio en que se vive, en el cual se establece la comunicación, en el que se desarrollan las actividades mas íntimas de las familias, es inapropiado...Es probable que las condiciones inapropiadas de los espacios en casas llamadas de interés social produzcan conductas mas agresivas y violentas, pues la incomodidad, la estrechez de los espacios, la falta de respuesta a un confort climático elemental, provoca cambios de conducta sustancialmente. (XXII CLEFA, 2007:309)

De esta forma los conceptos encerrados en el diseño habitacional en México, sus dimensiones, relación y ordenación de componentes espaciales, están referidos a normas y reglamentos que señalan requerimientos mínimos infrahumanos. 
De esta manera podemos concluir que las condiciones de convivencia en los espacios de la vivienda deben garantizar el desarrollo adecuado de los individuos, tanto desde el punto de vista formal como funcional, y esto, está estrechamente vinculado con la dimensión social del concepto de sostenibilidad integral, es decir, con el diseño sostenible. En el enfoque habitacional diseñar de forma sostenible implica que las viviendas edificadas en los espacios llamados lotes o terrenos deben ser adecuadas a las exigencias sociales, es decir, contener en su forma y función los requerimientos espaciales acordes a las necesidades del usuario en su evolución, de tal forma que el usuario logre apropiárselos otorgando facilidades y flexibilidad necesarios para su adecuación.

Entendiendo lo anterior el precedente sustentable, de acuerdo a Edwards Brian (2004) ${ }^{6}$ sugiere tres principales referentes: físico-espacial, sociocultural y económico. Por lo tanto, se considera que la transformación espacial está estrechamente ligada con el referente físico-espacial, dicha relación hace sugerir una retrospectiva analítica de los espacios que conforman a la vivienda que ha de garantizar el conocimiento racional de su conformación conceptual y de esta forma quizá proporcionar sugerencias hacia la evaluación de la misma.

Este artículo propone una herramienta denominada Instrumento de Análisis Gráfico Espacial, que en adelante denominaremos por sus siglas $I A G E$, cuya formulación forma parte de la Tesis de Maestria denominada: Estudio Tipológico de la Vivienda para la Clase Social Trabajadora Asalariada de Bajo Ingreso Económico: caso Poza Rica, Veracruz. Esta herramienta pretende abordar el análisis espacial a través de estrategias de tipo Cuantitativo estructurado por: a) el análisis del objeto o sitio de estudio con indicadores cuantificables incluidos en las respectivas variables; $b$ ) el análisis de las necesidades de los usuarios a través de la observación del espacio interior y su transformación. Al respecto, el análisis se basara en la transformación de la ordenación espacial entendida como parte de un todo (ciudad). Así, el contexto histórico y urbano de la vivienda, son vistos a través de ella y no al contrario, como tradicionalmente se ha abordado.

\footnotetext{
${ }^{6}$ Edwards, Brian y Hyett Paul. Guía Básica de la Sostenibilidad. Barcelona: Editorial Gustavo Gili, 2004. 1era. Edición, p. 121.
} 


\section{Algunos conceptos}

Para realizar el proceso analítico tomando como categoría de interés a la transformación espacial se requiere de una operación racional, para esto se recurre a la Tipología, como instrumento mental para el conocimiento racional abstracto.

De acuerdo a diversos autores como Guerrero Luis (1998) ${ }^{7}$, Martínez Caro (1990) ${ }^{8}$ y Martín Manuel $(1997)^{9}$ se deduce que la tipología es una operación dinámica traducida en la transformación, la yuxtaposición, la superposición y la articulación de elementos al objeto original distinguidas a través de la interpretación racional permitiendo deducir la esencia de un complejo de semejanzas o equivalencias de los rasgos generales que lo conforman. Explica un haz de relaciones por las que unos elementos arquitectónicos determinados se configuran en entidades arquitectónicas reconocibles. Se debe agregar que a partir del análisis racional se deduce la esencia constituyéndose esta por un concepto estructurado en un sistema acotado y definido por el que realiza la operación tipológica. Esta acción permite especificar los caracteres esenciales de cada elemento edificable, donde se plasman los requerimientos de cierto grupo de usuarios del pasado (social, cultural y económico). Para esto Aymonino agrega:

"La tipología significa un elemento indispensable para la proyectación, no tanto como método de análisis de las necesidades, como catalogo de prototipos que han definido y resuelto el esquema de necesidades". (BUSQUETS I., JOAN, 1999; 110)

Aymonino $(1973)^{10}$ especifica que la tipología es más que un análisis critico racional de las formas edilicias existentes cuyo único fin es conocer la evolución de los espacios, agrega que es un antecedente conceptual indispensable que se pueden tomar como referencia al realizar nuevas propuestas. Para esto es necesario catalogar los requerimientos sociales de cada grupo de usuarios.

En el estudio tipológico es posible conocer las características relevantes y distinguibles de una representación edificada surgiendo un patrón de reconocimiento basado en reglas y normas abstractas para evitar una confusión ante otros grupos o conjuntos edificados. Esta representación edificada es también llamada representación gráfica, ya que se basa en elementos de apoyo como: planos, cortes, fachadas, los cuales fueron resultado de la interpretación de necesidades físicas, biológicas y sociales de cierto grupo de usuarios.

La representación gráfica constituye un sustituto de la realidad considerándose como herramienta para lograr una interpretación semejante o parecida al espacio real representado. El análisis de la representación gráfica en la vivienda podría servir entre otros aspectos para reflexionar sobre aquéllos elementos espaciales que se han ido transformando a través del tiempo. Para expresar lo anterior se retoma lo dicho por Seguí:

\footnotetext{
${ }^{7}$ Guerrero Baca, Luís F. yRodriguez Viqueira, Manuel. Estudios de Tipología Arquitectónica. México D. F.: Ediciones Universidad Autónoma Metropolitana, 1998, 1era. Edición, p. 146.

${ }^{8}$ Martínez Caro, Carlos, De las Rivas, Juan Luís. Arquitectura Urbana. Madrid: Editorial Bellisco, 1990, 1ra. Edición.

${ }^{9}$ Martín Hernández, Manuel J. La Invención de la Arquitectura. Madrid: Ediciones Celeste, 1997, 1era. Edición, p. 231.

${ }^{10}$ AYMONINO, Carlo. La vivienda racional. Ponencias de los congresos. CIAM 1929-1930. Colección Arquitectura y

Crítica. Barcelona: Gustavo Gili, 1973, 1era. Edición, p.311.
} 
"Un proceso de descripción gráfica (de representación) puede entenderse motivado por el impulso o la necesidad de transcribir en un medio, un objeto y como condición de veracidad de la descripción...Y concluirá con un producto reconocible como una <<figuración>> del referente de partida. Cuando se representa algo gráficamente lo que no tienen duda es la entidad del referente, aunque siempre sea un problema qué es lo que queremos describir de él. Esto es la representación es también un proyecto ya que, para acometerla, hay que tantear y decidir un relato que englobe al referente, el momento, las propiedades a ser descritas y el sistema gráfico convencional en el que se va efectuar la consignación." (SEGUÍ, JAVIER, 2003;09)

Las descripciones gráficas, desde la reflexión, y análisis se convierten en objeto de investigación, en su posición de agente vinculado al proceso de generación de una propuesta a partir de un referente o Indicador, en este caso la transformación espacial.

Esta reflexión analítica lleva a la descomposición en los elementos constitutivos de las vistas gráficas analizadas (plantas, cortes, y fachadas arquitectónicos, así como fotografías y croquis) y la aproximación a los principios rectores de su génesis. Esto permite el estudio de los elementos de forma aislada y autónoma, estableciendo una relación formal y articulación entre ellos. Los procedimientos, de análisis y síntesis, facilitarán la obtención de un profundo conocimiento del espacio estudiado.

Entonces se deduce que la tipología es una herramienta o instrumento activo basado en el análisis crítico racional de configuraciones (representaciones gráficas de edificaciones) que permite determinar un concepto descriptivo de objetos, en este caso la vivienda y realizar una crítica comparativa. Al identificar y sintetizar los rasgos más sobresalientes se conseguirá descifrar un código, el cual permitirá analizar cada elemento, considerando al espacio como campo de acción modificable. Debe ocuparse de encontrar similitudes entre edificios y espacios abiertos en lo aparentemente distinto y poder generar soluciones en el diseño en futuras propuestas.

Se entiende que la existencia del espacio esta relacionada con las actividades que en ella se realizan y con los objetos contenidos en él. Los objetos otorgan una imagen específica al espacio, pero también el espacio impone el tipo de objetos o elementos físicos de acuerdo al objetivo principal de la proyectación.

"Entendemos aquí por espacios los que son delimitados por paredes y techos o por edificios, es decir, por algo <<artificial >> y creado por el hombre, o todo refugio natural que funciona como nicho ecológico...los espacios tienen forma, dimensiones, necesidades ecológicas y factores formativos que dependen del clima, de las costumbres, de la organización social y de la cabeza de la gente. Todos concurren a su conformación. “(PUPPO, ERNESTO, 1999; 21)

Los espacios son lugares conformados por elementos materiales, pero reflejándose también aspectos particulares como clima, roles y status social del sitio o lugar con respecto a otros. Es 
importante hacer énfasis que cada lugar difiere de otro, por lo tanto se considera inadecuado imponer espacios similares en cada uno de ellos. A partir de la definición anterior, el espacio en la vivienda es aquél cuya forma se encuentra delimitado por elementos naturales o artificiales (muro, cubierta, relieves, etc.) donde el hombre realiza actividades diversas (leer, trabajar, estudiar, hacer deporte, etc.). Se apropia de él cuando logra controlarlo a través de la representación de su esencia cuya forma y dimensión corresponda a sus necesidades físicas, biológicas y culturales.

Los espacios en la vivienda se encuentran en constante transformación, la cual se establece como categoría principal del referente físico-espacial de la sustentabilidad que ha de mantenerse ligada a los requerimientos sociales. El Diccionario Enciclopédico llustrado de la Lengua Española define a la transformación como: "hacer cambiar de forma. Transmutar, convertir una cosa en otra. Hacer mudar de porte, de conducta o de costumbres a algo a alguien...Según Valenzuela Carolina:

En el caso de la vivienda transformar implica re-crear, porque se trata de un cambio permanente que tiene que ver con la adecuación de la casa a sus habitantes. La evolución del hábitat privado es una característica intrínseca a la especie humana: como primera capa de relación con el entorno, la vivienda es el medio donde el hombre posee mayor poder de intervención" (VALENZUELA, CAROLINA, 2004; 74)

\section{Construcción del Instrumento de Análisis Gráfico Espacial-IAGE}

Para la construcción de la herramienta gráfica IAGE con la que se pretende abordar el estudio de la transformación espacial, se recurrió al análisis de factores emanados de algunos estudios que consideramos pertinentes desde su abordaje y destacados en su propuesta, que apoyaron la definición de variables y la selección de indicadores.

Hemos considerado necesario realizar un breve repaso de los autores y estudios mencionados para aclarar variables e indicadores que se manejan en el IAGE.

Respecto del acercamiento teórico al análisis tipológico tomamos las reflexiones de Martín Hernandez (1997) ${ }^{11}$, Aymonimo y Rossi (1999) ${ }^{12}$.

Manuel Martín Hernández $(1997)^{13}$ establece que para realizar un análisis tipológico es preciso que el especialista acote criterios orientados a conocer el entorno construido. Clasifica dos niveles tipológicos: el estratigráfico, es decir la relación entre los edificios y el contexto; y el compositivo, donde sitúa los criterios de uso, constructivos, geométricos, estilístico-formales o espaciales, referidos a la totalidad edificatoria.

En el IAGE, hemos propuesto una variable que denominamos forma-funcional en la que se vierten indicadores para analizar los niveles estratigráfico y compositivo descritos por Martín,

\footnotetext{
${ }^{11}$ Martín Hernández, Manuel J. La Invención de la Arquitectura. Madrid: Ediciones Celeste, 1997, 1era. Edición, p. 231.

${ }^{12}$ Busquets i Grau, Joan. La Urbanización Marginal. Barcelona: UPC (Ediciones de la Universidad Politécnica de Catalunya), 1999, 1era. Edición, p. 112.

${ }^{13}$ Martín Hernández, Manuel J. La Invención de la Arquitectura. Madrid: Ediciones Celeste, 1997, 1era. Edición, p. 231.
} 
con la intención de considerar la relación de la vivienda como parte de un todo (ciudad). En resumen los elementos compositivos que establece Martin se retomarán como variables gráficas: formal y funcional.

En estas variables se incluyó un apartado de antecedentes históricos, siguiendo a Rossi y Aymonino, como apoyo a la contextualización de la tipología analizada. Con relación a esto Aymonino (1999) define el análisis como "el estudio de los elementos organizativosestructurales artificiales...teniendo como finalidad su clasificación respecto a la forma urbana en un determinado periodo histórico"14, es decir, se enfoca más al aspecto funcional que al formal, cuando el análisis tiene un objetivo cognoscitivo. También se agrega lo afirmado por Rossi respecto "el análisis de la génesis de la forma (génesis que en muchos casos no existe) sino el análisis de cómo esta es entendida y viene asumida. De ahí que la relación entre tipo y forma no sea nunca mecánica..."15

Hasta aquí se ha deducido que las variables funcionales y formales son indispensables para tener un conocimiento más amplio de lo que se estudia, y como dice Manuel Martín (1997) ${ }^{16}$, el que analiza seleccionará los criterios de referencia. Es claro que los criterios se eligieron de acuerdo al tema o problemática abordada, es este caso la transformación espacial (interior y exterior).

Para el desarrollo de las indicadores operacionales del IAGE se recurre a la aplicación de estrategias de desarrollo conceptual en conjunción con elementos gráficos, por lo tanto las aportaciones de Luís F. Guerrero Baca y Rodríguez Viqueira (1998) ${ }^{17}$, Francis D. K., Ching $(2004)^{18}$, Paola Coppola Pignatelli $(1997)^{19}$, Eduardo López Moreno $(1996)^{20}$ y Busquet Joan $(1999)^{21}$ se consideran como los modelos de apoyo para el desarrollo operacional gráfico del objeto de estudio.

Lo especificado por Aymomino y Rossi $(1999)^{22}$ se encuentra reforzado con los estudios tipológicos expuestos por Guerrero Baca $(1998)^{23}$, pero en este caso los dos criterios de análisis, formales y funcionales, se estudian sin prejuicios deterministas. Esta integración no significa que se puedan combinar dichas variables durante su aplicación operacional en el caso de estudio elegido, simplemente que una no puede darse sin la otra, ya que cada una contiene su propios indicadores de análisis en conjunción con los elementos gráficos. Por lo tanto, para

\footnotetext{
${ }^{14}$ Busquets i Grau, Joan. La Urbanización Marginal. Barcelona: UPC (Ediciones de la Universidad Politécnica de Catalunya), 1999, 1era. Edición, p. 112

${ }_{15}$ Ídem.

${ }^{16}$ Martín Hernández, Manuel J. La Invención de la Arquitectura. Madrid: Ediciones Celeste, 1997, 1era. Edición, p. 231.

${ }^{17}$ Guerrero Baca, Luís F. y Rodríguez Viqueira, Manuel. Estudios de Tipología Arquitectónica. México D. F.: Ediciones Universidad Autónoma Metropolitana, 1998, 1era. Edición, p. 146.

${ }^{18}$ Ching, D. K., Francis. Arquitectura. Forma, Espacio y Orden. Barcelona: Gustavo Gili, 2004, 4ta. Edición, p. 398.

${ }^{19}$ Coppola Pignatelli, Paola. Análisis y Diseño de los Espacios que Habitamos. México D. F.: Editorial Árbol, 1997, 1era. Edición, p. 277.

${ }^{20}$ López Moreno, Eduardo. La vivienda Social: una historia. México: Programa Editorial Red Nacional de Investigación Urbana en coedición con Centro de Estudios Metropolitanos, Centro Universitario de Arte, Arquitectura y Diseño, en coedición con ORSTOM, 1996. 1era. Edición, p. 507.

${ }^{21}$ Busquets i Grau, Joan. La Urbanización Marginal. 1ª. Ed. Barcelona: UPC (Ediciones de la Universidad Politécnica de Catalunya), 1999, 1era. Edición, p. 112.

${ }^{22}$ Idem.

${ }^{23}$ Guerrero Baca, Luís F. and Rodriguez Viqueira, Manuel. Estudios de Tipología Arquitectónica. México D. F.:

Ediciones Universidad Autónoma Metropolitana, 1998, 1era. Edición, p. 146.
} 
efectuar la sincronización conceptual con los dispositivos gráficos es indispensable realizar disertaciones deductivas en su elección para conjuntarlos de manera lógica con su intensión interpretativa operacional. Finalmente las herramientas gráficas conceptuales o indicadores se integran razonablemente en las variables correspondientes del IAGE.

Es importante especificar que Luís F. Guerrero Baca y Rodríguez Viqueira (1998) ${ }^{24}$, ofrecen una guía de indicadores analíticos resultando propios para la presente investigación las siguientes: aspectos dimensionales y relacionales, condiciones de la ubicación, materiales y tecnología, sistemas de ordenamiento, voluntad expresiva principalmente.

Francis D. K., Ching (2004) ${ }^{25}$ ofrece una visión general de los elementos básicos, sistemas y órdenes que constituyen cualquier trabajo físico en el marco arquitectónico, los cuales son interdependientes, a fin de formar un conjunto integrado. Se refiere a la manera de disponer o coordinar los elementos y partes de una composición gráfica para producir una imagen coherente sugiriendo la referencia a la estructura interna, al exterior y al principio que confiere unidad al todo conteniendo diversas propiedades visuales como el contorno, el tamaño (longitud, anchura y profundidad), posición y orientación en concordancia con las variables y sus respectivos indicadores. También menciona el perfil o arista perimetral de un plano o de la arista de un volumen, dando relevancia a los de: planos (muros y cubiertas), las aberturas (puertas y ventanas) y las siluetas constructivas como elementos compositivos de los indicadores que conforman a las variables formal y funcional.

En conclusión, los principios gráficos expuestos permitieron personalizar el desarrollo de los elementos conceptuales implicados en la ordenación y organización de los espacios interiores y exteriores de la vivienda, los cuales se deducen con la aplicación del IAGE según corresponda a su intensión cognoscitiva referencial formal y funcional. Como se puede notar, el desarrollo de la metodología de análisis de intervención cuantitativa gira sobre los modelos gráficos derivándose el esquema general del Instrumento de Análisis Gráfico Espacial-IAGE como elemento operacional en la generación de nuevos procedimientos de intervención.

Para intervenir en el análisis espacial de la vivienda es preciso realizar una clasificación clara de las diversas áreas que conforman al sistema habitacional, por lo que se recurre a la clasificación realizada por Paola Coppola Pignatelli $(1997)^{26}$ cuyo esquema se sintetiza de la siguiente manera: privado individual (1-2 personas, recámaras, estudio, baño, armarios y cajones), privado Familiar (estancia, comedor, cocina, vestíbulo y closet), semiprivado (5 a 20 núcleos familiares, cuarto de obvies, lavadora y secadora, conectivo de circulación), semipúblico (50-200 núcleos familiares, estacionamiento, zona verde, conectivo de distribución -escaleras, balcones, espacios comunes). Así mismo hace énfasis en los elementos delimitantes del espacio clasificándolas en dos tipos: verticales (muros en sus diversas modalidades-de carga, de separación, de filtro de aire, de filtro de luz, de filtro visual, movibles y simbólica) y horizontales (piso y cubierta).

\footnotetext{
${ }^{24}$ Guerrero Baca, Luís F. y Rodríguez Viqueira, Manuel. Estudios de Tipología Arquitectónica. México D. F.: Ediciones Universidad Autónoma Metropolitana, 1998, 1era. Edición, p. 146.

${ }^{25}$ Ching, D. K., Francis. Arquitectura. Forma, Espacio y Orden. Barcelona: Gustavo Gili, 2004, 4ta. Edición, p. 398.

${ }^{26}$ Coppola Pignatelli, Paola. Análisis y Diseño de los Espacios que Habitamos. México D. F.: Editorial Árbol, 1997, 1era. Edición, p. 277.
} 
Da relevancia a los estudios sobre la relación de la forma de un espacio y su modo de uso, considerando a estos referentes como principios deductivos de la calidad de los espacios desde el punto de vista descriptivo y analítico. Las áreas espaciales concretadas se constituyen como la herramienta física de intervención del IAGE en su desarrollo conceptual interpretativo.

Es también relevante recordar que las variables formal y funcional se analizan a partir de precedentes históricos, esta intervención mixta es abordada por Eduardo López Moreno $(1996)^{27}$ al realizar el estudio interpretativo de un sitio específico, la ciudad de Guadalajara, Jalisco bajo la perspectiva de vivienda de interés social, dividiéndola en 2 vertientes: una histórica analítica interpretativa de modelos locales de desarrollo, de los usuarios y formas de producción del espacio urbano basándose en aspectos económicos, sociales y políticos; la segunda se refiere a la transformación del uso del suelo y la vivienda, es decir a nivel gestión. De esta forma se puede entender de manera clara lo especificado por Rossi y Busquet $(1999)^{28}$ en cuanto al aspecto formal deduciéndose que los acontecimientos históricos, administrativos, jurídicos, económicos de la vida diaria de una población están estrechamente vinculados no solo con la forma sino también con la función, surgiendo una tercera variable, la Socio-política.

Busquet Joan (1999) ${ }^{29}$ establece que para realizar una intervención tipológica es necesario elegir algún elemento definidor como categoría de estudio, el cual deberá contener pretensiones de análisis para el conocimiento pleno de los parámetros o problemática de interés. Lo anteriormente dicho se complementa con la incorporación de diversos indicadores estableciendo principalmente las siguientes: descripción espacial por periodos, forma y tamaño de la parcela, mejoras internas, posición de la vivienda respecto al terreno, la distribución interna, la distribución externa, clasificación de lotes (estrecho, medio y amplio), y las etapas en el proceso de crecimiento según tipos de parcelación. Precisamente estas potencialidades se hacen presentes en el desarrollo de la actividad conceptual del problema y su traducción gráfica como elemento visual.

La presencia de la vivienda en el contexto de análisis que se elija es producto de diversos hechos históricos durante su evolución, de tal forma se considera que el origen de ciertas tipologías están relacionadas con los hechos sociopolíticos. La variable sociopolítica se integra como primera parte del Diseño del Instrumento Gráfico Espacial-IAGE como proceso descriptivo precedente de los diversos casos de estudio del sitio seleccionado.

Busquets i Grau, Joan $(1999)^{30}$, establece que las formas edilicias constituyen la reproducción social, las cuales han de reflejarse en la forma y función, en correspondencia a determinada etapa histórica y esquema tipológico. Entendiéndose este esquema como permanente dentro de un conjunto dinámico (morfología) y siempre precedido por acontecimientos relevantes. Se deduce entonces que los hechos o acontecimientos relevantes de determinado contexto están estrechamente ligados con la forma de los espacios y su utilidad y no entenderse estos como

\footnotetext{
${ }^{27}$ López Moreno, Eduardo. La vivienda Social: una historia. México: Programa Editorial Red Nacional de Investigación Urbana en coedición con Centro de Estudios Metropolitanos, Centro Universitario de Arte, Arquitectura y Diseño, en coedición con ORSTOM, 1996. 1era. Edición, p. 507.

${ }^{28}$ Busquets i Grau, Joan. La Urbanización Marginal. Barcelona: UPC (Ediciones de la Universidad Politécnica de Catalunya), 1999, 1era. Edición, p. 112.

${ }^{29}$ Idem.

${ }_{30}$ Idem
} 
un objeto plástico o escultural. Es así como se refuerza el indicador sociopolítico considerándose imprescindible en el proceso analítico de la vivienda.

Para realizar abordar el estudio tipológico es preciso establecer una relación conceptual de los principales elementos de análisis del IAGE establecido para su desarrollo.

Iñaki Ábalos (1995) $)^{31}$ expone una clasificación de los espacios constituyentes de la vivienda u hogar del ser humano anteponiéndola como el lugar de origen del comportamiento, entre ellas se encuentran: la estancia, el área de convite, el dormitorio, la cocina, baño y otros. Por lo tanto, sirvió de apoyo para definir las áreas interiores que se incluyen en la herramienta de análisis gráfico.

Seguí Javier (1996) ${ }^{32}$ permite vincular teóricamente a la vivienda dentro de la expresión gráfica, definiéndola como resultado de la expresión de la transcripción de un referente. Tomando como campo de representación al espacio, aquél como resultado de la configuración. Puppo Ernesto (1999) ${ }^{33}$ agrega que los espacios se encuentran limitados por elementos verticales y horizontales, otorgándole forma de acuerdo a cada organización social específica. A esto se le puede agregar lo especificado por Sola De Morales $(2002)^{34}$ y Pernas del Risco $(1999)^{35}$ sobre la evolución de los espacios a través del tiempo de acuerdo a las necesidades y costumbres.

De esta forma en su origen el IAGE pretende unificar diversos criterios que generalmente son abordados de manera particular, por lo tanto se plantean tres variables: socio-politica, funcional y formal. Es decir, se patentará un instrumento de análisis enriquecido por herramientas conceptuales y gráficas enfocado a la transformación espacial en la vivienda hacia el precedente sustentable espacial.

Este instrumento se aplicará a un sitio específico, Poza Rica Veracruz, en el cual los estudios tipológicos son nulos hasta la actualidad, por lo tanto el presente será la primera intervención tipológica tomando como base la unificación de criterios e indicadores establecidos por diversos especialistas en otros estudios pero de manera personalizada y evitando determinismos. Por tanto se pretende que dicho trabajo sea útil en estudios posteriores.

En conclusión, la sustentabilidad referida al espacio adecuado en relación a la transformación se propone abordarla a través de tres variables: la socio-política, a través de un Procedimiento Descriptivo- $P D$, el cual se estructurará por aspectos históricos e Institucionales, la variable funcional propone el Procedimiento Operativo-PO para fines cognoscitivos de los elementos organizativos estructurales-artificiales (términos utilizados por $\mathrm{C}$. Aymonino) de los espacios. En la variable formal se propone el Procedimiento Interpretativo-PI (término deducido de los principios compositivos de Manuel Martín), el cual sirve para analizar de cómo la forma es

\footnotetext{
${ }^{31}$ Ábalos, Iñaki. La buena vida. Visita guiada a las casas de la modernidad. Barcelona: Gustavo Gili, 2001, 2da. Edición, p. 195.

${ }^{32}$ Seguí, Javier, Planell Joaquín, Burgaleta Pedro M. La Interpretación de la Obra de Arte. Madrid: Editorial Complutense, 1996, 1era. Edición.

${ }^{33}$ Puppo, Ernesto. Un espacio para Vivir. México D. F.: Editorial Alfaomega, 1999, 1era. Edición.

${ }^{34}$ SOLA-MORALES. Introducción a la Arquitectura, Conceptos Fundamentales. México: Ediciones UPC, 2002.

${ }^{35}$ Pernas Del Risco, Orlando y otros. La Casa Unifamiliar. Colectivo de Autores Arq. Vladimir Duormanov. México D. F: Instituto Politécnico Nacional, 1999, 1era. Edición, p. 247.
} 
entendida y asumida en relación con acontecimientos trascendentes del contexto analizado permitiendo codificar principios de ordenación en la gran complejidad.

La categoría transformación espacial tiene como referente aquéllos estudios ejecutados por especialistas en tipologías, pero con la particularidad de contener la esencia experimental de causa y efecto acorde al factor sustentable basados en técnicas instrumentales operacionales, es decir de abstracciones compositivas de los levantamientos arquitectónicos y fotográficos.

Para evitar la simple abstracción geométrica se establece la conexión entre los sucesos reales con su ubicación espacial y cronológica dejando de ser solamente descriptivas, de esta forma los tres procedimientos resultan complementarios en al análisis de la sustentabilidad espacial. Con lo anterior se obtendrá la interpretación de cada uno de los prototipos de vivienda elegidos en cada caso de estudio del sitio en cuestión.

De acuerdo a lo especificado en el Instrumento de Análisis Gráfico Espacial-IAGE se puede percibir un apartado de indicadores, los cuales difieren en cada procedimiento estableciendo diversas técnicas instrumentales de medición que llevarán a la deducción o explicación de los fenómenos de estudio seleccionados con base a los criterios de interés especificados en la transformación espacial teniendo como referente a la sustentabilidad espacial en la vivienda.

\section{Desarrollo del Instrumento de Análisis Gráfico Espacial-IAGE}

Como se explicó en el apartado anterior, desde el punto de vista teórico conceptual y metodológico es posible aplicar propuestas de diversos especialistas que han surgido a lo largo del estudio de la vivienda como Guerrero, Baca, Manuel Martín, Joan i Busquet, López Moreno, entre otros, esto en las diversas etapas con respecto al espacio, es decir a partir del análisis tipológico de los distintos niveles hacia una retrospectiva espacial como referente hacia la evaluación de alternativas físico-espaciales. A continuación se presenta en al cuadro 2 la deducción sintética de los elementos de estudio del espacio en la vivienda y sus diversos aspectos de análisis:

\begin{tabular}{|c|c|}
\hline \multicolumn{2}{|c|}{ CUADRO 2.Desarrollo Sintético del Instrumento de Análisis Gráfico Espacial-IAGE } \\
\hline & Línea de Investigación: Tipologías. \\
\hline & Categoría: Transformación espacial en la Vivienda \\
\hline $\begin{array}{l}\text { Variable Socio- } \\
\text { política: }\end{array}$ & $\begin{array}{ll}\text { - } & \text { Eventos detonantes del desarrollo de la Vivienda. } \\
\text { - } & \text { Estruidades económicas predominantes. } \\
& \text { Estructurídica. }\end{array}$ \\
\hline Variable Funcional: & $\begin{array}{l}\text { Indicadores Deductivos abordados a través de técnicas Instrumentales: } \\
\text { Contexto Urbano } \\
\text { 1. Contexto Urbano o emplazamiento General:. } \\
\text { 1.1 Equipamientos Urbana del Conjunto Habitacional: } \\
\text { - Ejes peatonales (Sendas) (andadores callejones). } \\
\text { - Espacio recreativo (canchas y campos deportivos, parques, juegos infantiles, etc.). } \\
\text { - Espacios educativos (de todos los niveles: preescolar, básica, media superior, etc.). } \\
\text { - Espacios religiosos: iglesia, capilla. } \\
\text { 1.2 Levantamiento Urbano del Conjunto Habitacional } \\
\text { 1.3 Servicios de abastecimiento en el Conjunto Habitacional. } \\
\text { - Disposición y Jerarquía de Vialidades Principales (nombre y medidas-ancho). } \\
\text { - Vialidades Secundarias (nombre y medidas-ancho). } \\
\text { 1.4 Superfión con el entorno. } \\
\text { 1.5 Trazado: del conjunto habitacional } \\
\text { - Parrilla rectangular } \\
\text { - } \text { Radial }\end{array}$ \\
\hline
\end{tabular}




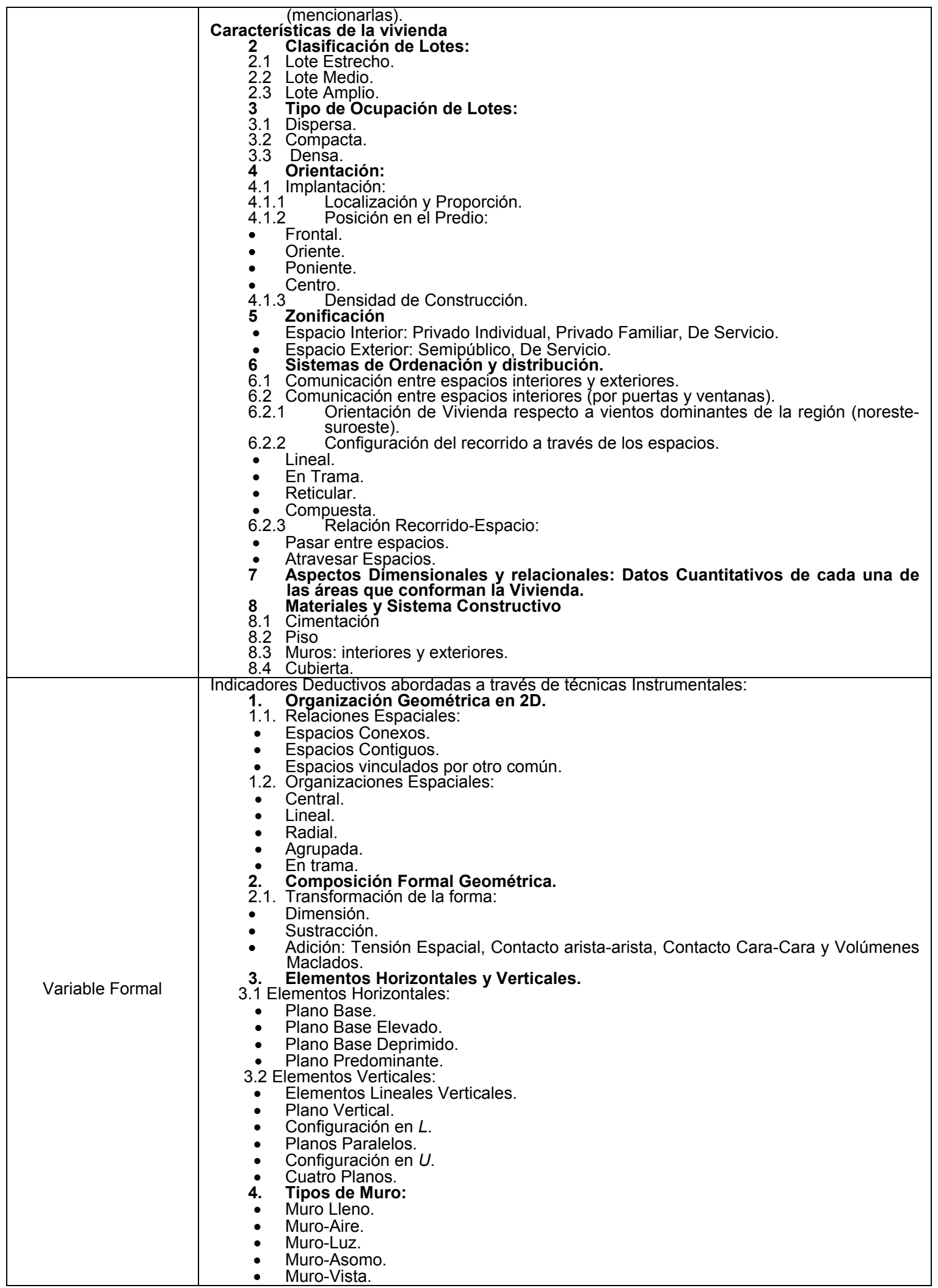


Se considera que los diversos estudios realizados por los especialistas en materia de vivienda mencionados en la presente investigación son precisos en su desarrollo, sin embargo se consideró relevante producir una estrategia de aplicación para deducir el origen de cada elemento de estudio, es decir un instrumento gráfico como elemento de abstracción mental y figurativo conceptual. Durante su aplicación en el caso de estudio, dicho instrumento funciona simultáneamente como fragmentador y organizador de los elementos o espacios que conforman a la vivienda: el instrumento como rol fragmentador interviene a partir de los segmentos implicados en la composición y organización del contexto espacial urbano hasta los elementos que estructuran el ámbito interior espacial de la vivienda. Estas delimitaciones de estudio permiten establecer un concepto organizador del conjunto de ideas que llevan a la conformación de los elementos gráficos de apoyo para la generación y relación de cada segmento gráfico conceptual con la realidad.

En la modalidad de producción de conocimiento se emplea como elemento básico de análisis a laa composiciones gráficos, cuya finalidad metodológica precisa en proponer y realizar un esquema dinámico y menos austero que ha de permitir en el transcurso de su desarrollo mantener una secuencia lógica expresiva en sincronización con un lenguaje claro y conveniente para la descripción formal y funcional. Este proceso ordenado, clasificado y analizado impide confusiones en el desarrollo de la etapa descriptiva de las variables y sus respectivos indicadores establecidos de manera estratégica en el IAGE. De tal forma que en el análisis final comparativo de la transformación espacial se ejecuta el maclaje de las descripciones componentes de cada variable que ha de facilitar y otorgar los elementos de apoyo para realizar afirmaciones deductivas referidas a la problemática espacial que se manifiesta en las viviendas del caso de estudio.

El esquema permite aplicar de manera independiente en el estudio del caso seleccionado los indicadores respectivos que permiten conocer la transformación espacial formal desde los ámbitos básicos hasta los más complejos en su desarrollo gráfico-conceptual. Análogamente sucede con la variable funcional, donde los indicadores desempeñan como elementos de conocimiento gráfico-conceptual permitiendo evaluar la funcionalidad de los espacios de los diversos ámbitos exteriores e interiores. Es bien sabido que en la producción de viviendas sobre todo aquélla destinada a los usuarios de bajo ingreso, generalmente se hace caso omiso de las normas y reglamentos de diseño decretadas por diversas instancias gubernamentales y organismos institucionales, por lo tanto este esquema permite comprobar de manera simultánea las referencias gráficas y cuantitativas en la evaluación de los espacios y de esta forma afirmar de manera justificada el problema de inadecuación de la disposición de los espacios que conforman el contexto urbano y el espacio que contiene la vivienda, es decir del referente físico-espacial.

En el proceso de diseño de este instrumento gráfico siempre se tuvo como herramienta protagonista en su desarrollo a la expresión gráfica, entendida como la codificación mental a través de elementos visuales, en este caso la interpretación de las necesidades de los usuarios en cada época o caso de estudio en la vivienda y su contexto. Esta codificación se hace evidente a través de planos, fotografías, croquis, imágenes entre otros, es decir, de las interpretaciones de las necesidades del usuario. Así, también es importante mencionar que durante la recopilación de información para su aplicación en el caso de estudio elegido, se debe recurrir a fuentes bibliográficas actualizadas y pertinentes al IAGE. En varias ocasiones 
las fuentes de datos se encuentran ubicadas en lugares ajenos al sitio de estudio, por lo tanto también es importante consultar a archivos históricos, instancias gubernamentales, bibliotecas entre otros.

También se considera importante la participación de los habitantes de las viviendas analizadas y de personas conocedoras de la historia de los casos de estudio Por lo tanto, la estructuración del IAGE promueve la interacción entre factores, instancias y protagonistas externos e internos del sitio de estudio resultando enriquecedor en la modulación y producción de conocimiento. Al conocer la realidad de manera racional se pretende conocer, analizar y sugerir alternativas durante el proceso de abstracción mental gráfico-conceptual, por lo tanto el perfeccionamiento de su proceso constructivo y desarrollo se dio gradualmente intercambiando elementos gráficos y conceptuales.

\subsection{Sobre su aplicación}

El Instrumento de Análisis Gráfico Espacial-IAGE se constituye una alternativa gráfica racional de abstracción mental para abordar el referente de estudio físico-espacial, el cual forma parte de uno de los preceptos de la sustentabilidad en la vivienda. De esta forma se tiene como categoría de proceso a la transformación espacial de la vivienda partiendo de su contexto hasta las áreas interiores que la conforman, por lo tanto esta herramienta se considera aplicable en cualquier sitio independientemente de su clima, ubicación geográfica, topología entre otros, ya que precisamente las características particulares de cada sitio son las que se ordenarán y clasificarán en los módulos de análisis que ofrece el IAGE, es decir, en sus respectivas variables e indicadores, con la pretensión de realizar una descripción sistemática y posteriormente someterlo a un análisis comparativo. En cuanto al criterio temporal, se propone que este sea acotado a partir de hechos relevantes, ya que así será posible identificar con mayor facilidad los elementos que se contraponen o alinean en el patrón se análisis de cada periodo delimitado.

Para realizar el análisis funcional existen normas y reglamentos oficiales sobre las especificaciones mínimas superficiales de los componentes espaciales interiores y exteriores de la vivienda en relación con el usuario y sus características corporales con sus respectivas funciones simples y complejas. Por lo tanto en la crítica comparativa donde se evidencia la presencia de referencias cuantitativas es preciso contextualizarlas a especificaciones provenientes de fuentes informativas gubernamentales, aunque es importante aclarar que estas referencias dimensionales varían en relación con la constitución física del usuario, ya que en el presente estudio las especificaciones oficiales están contextualizadas para la población de origen mexicano cuyo referente proyectual se tiene a especificaciones de Plazola falta año.

En la presente investigación la estructuración y aplicación del IAGE como instrumento referencial cuantitativo se considera como alternativa de análisis gráfico-conceptual para conocer, describir y analizar las problemáticas espaciales a las que se enfrenta la población vulnerable, aquélla que invierte sus reducidos ingresos en la vivienda, aquélla regularmente gestionada a través de programas e instancias gubernamentales, que aunque pretenden aplicar normativas y principios hacia la vivienda adecuada dichos preceptos son corrompidos durante el proceso de ejecución. 
Durante su aplicación permite interrelacionar a la vivienda como elemento referencial básico arquitectónico con los requerimientos y hechos sociales, cuyo proceso de análisis interviene directamente sobre espacios reales de la vivienda. Dicho proceso interactivo en su aplicación de análisis permite discernir racionalmente el reconocimiento de la situación espacial formal y funcional para realizar proposiciones sobre la adecuación de la vivienda en sus criterios espaciales en su respectivo contexto sociopolítico. Lo anterior desarrollado a través de una estrategia mental tipológica como fundamento racional de reconocimiento, lo cual podría servir de fundamento para estudios y problemáticas posteriores.

En la aplicación del IAGE se evitan determinismos con la intensión de realizar un estudio holístico espacial que permite el conocimiento pleno de la conceptualización de los espacios a través de acciones físicas evidentes en la apropiación por parte del usuario. Lo anterior nos llevará a deducir los efectos negativos o positivos de dichas acciones que se establecen como referentes evidenciales de la vivienda adecuada. Esta apropiación lleva implícito la flexibilidad de cada espacio para su modificación durante las etapas de vida del usuario.

Para su aplicación es necesario que el objetivo de estudio corresponda a la problemática abordada en la presente investigación, es decir en torno a los espacios adecuados y su transformación. El sitio de estudio debe ser conocido en su totalidad así como también los casos de estudio para evitar confusiones en su descripción y análisis comparativo final.

Se considera que su aplicación es de gran ayuda en diversas líneas de investigación relacionadas con la vivienda desde enfoques culturales, operacionales, y de evaluación.

El enfoque cultural es proyectado en el IAGE, ya que al abordar el aspecto sociopolítico permite indagar sobre los hechos históricos relevantes en la evolución del sitio de estudio que se elije, estos hechos están estrechamente relacionados con los aspectos sociales y económicos que indudablemente influyen en la generación de cierto tipo de arquitectura habitacional siempre en concordancia con las necesidades de los usuarios en cuestión. Por ende se deduce que la función de los elementos espaciales de la vivienda estan indudablemente relacionadas con las características del lugar y sus usuarios, es decir, derivadas a partir de costumbres, formas de ser o actividades generadas por la forma de vida de los pobladores en cierto contexto estrechamente relacionado con aspectos políticos, económicos y sociales. Por lo tanto la descripción sociopolítica de la primera fase del instrumento es un módulo que permite el reconocimiento cultural, pero dejando claro que solo de manera referencial, ya que para un mayor conocimientos es necesario la aplicación de estudios más exhaustivos, quizá de tipo cualitativo donde sea necesario una intervención interdisciplinaria con la participación de sociólogos, antropólogos, ecónomos, etc.

En la sección descriptiva formal y funcional de la herramienta operacional que antecede al análisis crítico se considera aplicable a cualquier tipo de edificación, ya que los elementos gráficos permiten desengranar los elementos que conforman al espacio arquitectónico y al contexto urbano como referente. De esta forma se efectúa una descripción gráfica pertinente al lenguaje arquitectónico que ha de permitir evitar confusiones en su interpretación gráficoconceptual, ya que esto es de vital importancia en el análisis comparativo. Con la respectiva descripción se hacen evidentes las configuraciones espaciales a través de esquemas y 
gráficos, los cuales prescriben un código de reflexión e interpretación sistemática en un patrón de reconocimiento.

El IAGE como elemento evaluador de los espacios toma como criterio de análisis la adecuación espacial en la vivienda, por lo que dicha herramienta permite desmenuzar los aspectos que atañen al desarrollo de los elementos espaciales exteriores e interiores de la vivienda, De esta forma se efectúa una separación racional de los aspectos formales, funcionales y sociopolíticos realizando una descripción independiente de cada una para posteriormente realizar una interpretación donde convergen los tres anteriores y evitar deducciones plásticas. Es decir, no se queda en el aspecto descriptivo sino que es analítico a través de una comparación crítica de los casos de estudio y en sus diversos periodos.

En deducción, su aplicación como elemento evaluador permite establecer proposiciones sobre la disponibilidad cuantitativa de los espacios en la vivienda enfocada a la denunciación e intervención pronta sobre las instancias implicadas en dicha inadecuación, ya que finalmente afectan al usuario que es vulnerable ante las acciones clandestinas de los constructores y encargados de su gestión produciendo la deformación de los principios establecidos por los Objetivos del Milenio en conjunción con la agenda Hábitat. De esta manera como evaluador espacial es de vital importancia desde el punto de vista cuantitativo.

\subsection{Sobre sus limitaciones}

EI IAGE es un instrumento que en su desarrollo requiere de la acotación temporal y espacial para su aplicación, ya que por presentar indicadores que requieren conocimiento amplio de hechos internacionales, nacionales, regionales y locales, es preciso comprender periodos cortos para obtener la descripción plena que permita realizar una interpretación comparativa racional exhaustiva entre las tres variables, formal, funcional y socio-política., de lo contrario la interpretación resultaría irrelevante para aportaciones cognoscitivas y propositivas referidas a la adecuación espacial.

Precisa en el conocimiento de transformación social de un sitio específico a partir de la arquitectura, conocer la evolución a través de ella y no a la viceversa, a cuya referencia denominamos transformación espacial. Así en el proceso de análisis de dicha categoría es importante la recolección de parámetros medibles y cuyo contenido sea comprobable de manera objetiva a través de indicadores racionales de tal forma que se evade el estudio del aspecto sociológico, el cual como se sabe comprende parámetros perceptivos e intuitivos de los usuarios o protagonistas de la vivienda, por lo tanto de enfoque subjetivo.

El aspecto subjetivo se traduce en una metodología cualitativa, es decir una investigación basada en elementos no medibles como la percepción del espacio, el cual está estrechamente relacionado con las forma de ser del individuo, la identidad, y aspectos psicológicos en concordancia con el contexto en que se desenvuelve, es decir, costumbres, valores, emociones, cultura etc., haciéndose evidente en la forma de concebir el espacio y de apropiación durante la permanencia del usuario es cuestión. Donde el significado del espacio resulta expansivo y fundamentado principalmente en la experiencia y la intuición, que deriva generalmente de puntos de vista particulares de los usuarios implicados en el desarrollo de la investigación. 
Para aplicar el mencionado enfoque es preciso efectuar estudios de intervención directa con los habitantes de las viviendas con la aplicación de entrevistas, test, precedidas de intervenciones altamente especializadas, donde quizá intervengan diversos expertos como sociólogos, psicólogos, ecónomos, etc. concluyendo en un estudio multidisciplinario con la finalidad de abordar la problemática de manera profunda que ha de evitar interpretaciones erradas o someras en la conceptualización de adecuación de los espacios.

Deduciblemente el enfoque cualitativo resulta enriquecedor porque proporciona datos que van más allá de lo medible, es decir proporciona elementos perceptivos que otorgan valor a la configuración espacial independientemente de los parámetros establecidos como correctos. Esto es, por ejemplo, la vivienda de interés social está diseñada para aquéllos trabajadores asalariados de bajo ingreso supuestamente comprendiendo en su configuración espacial parámetros mínimos para la correcta ejecución de las actividades propias de subsistencia. Esta vivienda es adaptable a los usuarios que habitan en las ciudades, donde el uso del suelo es reducido, y donde la gente se ha acostumbrado a espacios comprimidos para la realización de sus actividades cotidianas básicas, como dormir, comer, cocinar, lavar, etc. De tal forma que si dichas viviendas se trasladan a un medio rural, seguramente la concepción espacial de los usuarios de estos lugares difiere de los usuarios citadinos resultando para ellos espacios impropios por sus dimensiones comprimidas aunque técnicamente sean adecuados. Esto es porque la gente de la zona rural esta acostumbrada a convivir en espacios abiertos y dimensiones extensas debido a la accesibilidad en el uso del suelo en los lugares rurales.

Con el ejemplo anterior se quiere demostrar que el aspecto cualitativo es importante para realizar un estudio espacial en la vivienda porque proporciona deducciones obtenidos de aquéllos usuarios que coexisten directamente en la configuración espacial y que indudablemente permiten deducir concepciones adecuadas sobre el uso y percepción de los espacios. Esto no significa que el método cuantitativo sea inválido, al contrario los dos enfoques son indispensables para el estudio de la adecuación espacial de la vivienda. Esta adecuación retomada y vinculada con el indicador área suficiente para vivir en sus diversos ámbitos conceptuales o indicadores de cada variable según sea el caso. Sin embargo la presente investigación, la cual se encuentra en proceso de construcción, solo se enfoca en el aspecto objetivo quedando abierto a la incorporación posterior del enfoque subjetivo, que como se dijo implica estudios especializados de convergencia multidisciplinar.

Se pretende que el Instrumento de Análisis Gráfico Espacial-IAGE sea completado en estudios posteriores con la incorporación de una herramienta cualitativa cuyo enfoque sea subjetivo. Así se tendría la posibilidad de proponer y realizar un análisis comparativo entre estas dos vertientes lo que implicaría nuevas deducciones del espacio resultando más enriquecedor en la evaluación del indicador denominado adecuación espacial en los espacios interiores y exteriores de la vivienda, sobre todo aquélla destinada a la población vulnerable por presentar mayores inconvenientes en su ejecución. 


\section{Comentarios finales}

El derecho a la vivienda siempre será necesidad primaria del ser humano, ya que le proporciona seguridad social, por esto se considera primordial ofrecer una vivienda adecuada a la población vulnerable, es decir aquélla de bajo ingreso económico. Por ello se especula que es necesario realizar instrumentos científicos para la evaluación de la vivienda, estrechamente relacionados con los referentes sustentables, en este caso se aborda a partir del físico-espacial estrechamente vinculado con el indicador área suficiente para vivir, y ambas establecidas como preceptos de la vivienda adecuada. Así se considera que la sustentabilidad en la urbe puede ser lograda empezando por las unidades que la conforman, es este caso, se propone a la vivienda, ya que es el refugio del ser humano e indispensable para su desarrollo personal reflejado directamente en los grupos sociales en los que interactúa.

De esta forma se propone un modelo operacional de enfoque cuantitativo, al cual denominamos Instrumento de Análisis Gráfico Espacial-IAGE, dicho instrumento se aborda a partir de la línea de investigación tipológica como herramienta racional abstracta. Por ende se establece a la transformación espacial como categoría de análisis teniendo como precedente de evaluación a la vivienda adecuada acotada en su indicador área suficiente para vivir. Para esta evaluación se proponen parámetros medibles tomando como referencia a especificaciones establecidas por instancias oficiales en relación con los espacios mínimos. Así se deducen los aciertos y desaciertos de la transformación de la vivienda en un sitio específico.

En dicha categoría de análisis de proponen las variables formal, funcional y sociopolítico, los cuales se desarrollan a partir de indicadores tomando como herramienta operacional a la expresión gráfica. Es importante mencionar que el IAGE se encuentra en proceso de diseño para su perfeccionamiento, de tal forma que se somete a aplicación continua a un caso de estudio seleccionado a prueba de ensayo y error considerándose una metodología de intervención directa en el proceso de construcción. En dicho proceso convergen datos obtenidos a través de acervos bibliográficos y levantamientos en los casos de estudio del sitio elegido. Al final se pretende realizar la sincronización racional de las variables que se traducirá en una interpretación analítica comparativa, detonándose esta como elemento evaluador de la adecuación espacial de la vivienda.

Las aplicaciones mencionadas se han deducido tomando como parámetro el nivel de avance del diseño y aplicación del IAGE en un sitio de estudio, en este caso en Poza Rica, Veracruz de la República Mexicana, por lo tanto se considera pertinente mencionarlas sin dejar a duda su veracidad deductiva. Estas aplicaciones se reducen en tres enfoques culturales, operacionales, y de evaluación consideradas complementarias en su interpretación conjunta.

Teniendo conocimiento previo de la utilización y evolución espacial se obtuvo referentes cuantitativos, los cuales permitieron comprobar objetivamente las características formales y funcionales estrechamente ligados con hechos sociopolíticos. Si esta herramienta se aplica estratégicamente a conjuntos habitacionales proponiendo periodos sincronizados permitiría la construcción de un patrón modelo evolutivo más amplio. Este último llevaría al reconocimiento de las características acertadas y finalmente la generación de principios espaciales adecuados 
en el sitio de estudio. Por tal motivo las tres variables interactúan simultáneamente en la transformación espacial, como precedente sustentable.

Las limitaciones señaladas corresponden a alcances separados en la presente investigación, por lo tanto se considera que esta herramienta es susceptible de indexación del enfoque cualitativo, que como se explicó es importante para definir de manera completa el significado espacial, por lo tanto las divergencias cuantitativas y cualitativas pueden ser unificadas a través de procesos mas especializados

El procedimiento desarrollado en la presente investigación forma parte de una sección de la tesis de maestría en la que se ha propuesto casos de estudios específicos de un sitio seleccionado, Poza Rica ,Veracruz, pero dicha aplicación es omitida debido a la considerable extensión, ya que tampoco se considera conveniente presentar una aplicación sintética porque como se ha hecho explicado se toma como base operacional a la herramienta gráfica traduciéndose en esquemas extensivos, los cuales no pueden ser evadidos en su presentación. Pero se debe tener la certeza que su aplicación ha sido sometida a diversas etapas de prueba en el proceso de diseño y aplicación del IAGE. 


\section{Bibliografía}

1. Busquets i Grau, Joan. La Urbanización Marginal. Barcelona: UPC. Ediciones de la Universidad Politécnica de Catalunya, 1999 1era. Edición, p. 254.

2. Edwards, Brian y Hyett Paul. Guía Básica de la Sostenibilidad. Barcelona: Editorial Gustavo Gili, 2004. 1era. Edición, p. 121

3. XXII Conferencia Latinoamericana de Escuelas y Facultades de Arquitectura. Retos de la Formación e Identidad en Diseño y Arquitectura en el Marco de la Globalización. Ciudad de la Antigua, Guatemala, Guatemala. 5-9 Noviembre 2007. P. 669.

4. Puppo, Ernesto. Un espacio para Vivir. México D. F.: Editorial Alfaomega, 1999, 1era Edición.

5. Seguí, Javier, Planell Joaquín, Burgaleta Pedro M. La Interpretación de la Obra de Art. Madrid: Complutense Publishing, 1996, 1era. Edición, p. 324

6. Suárez, Francisco. Derechos Económicos, Sociales y Culturales en América Latina: su situación actual. Comisión Económica para América Latina y el Caribe (CEPAL), Naciones Unidas, 2001. (en línea). 2001- (citado en 15 febrero 2007), p. 82. Disponible en Internet: http://www.ugr.es/ filode/pdf/contenido35 3.pdf.

7. Organización de la Naciones Unidas. Los Objetivos del Desarrollo del Milenio en México (en línea). México D. F.: gabinete de Desarrollo Humano y Social/Comisión Intersecretarial de Desarrollo Social, 2006-(citado 7 julio 2007). Disponible en Internet: http://www.onu.org.mx.

8. Valenzuela, Carolina. Plantas transfromables: La vivienda colectiva como objeto de intervención.Arq. (Santiago). (en línea). Diciembre 2004, no.58 (citado 10 mayo 2007), p. 74-77. Disponible en Internet: http://www.scielo.cl/scielo.php?pid=S0717$\underline{699620040058000022 \& \text { script }=\text { sci arttext. }}$. 
Architecture, City, and Environment

Arquitectura, Ciudad y Entorno 